\title{
GIXA is a new structural member of the radical copper oxidase family and is required for glycan deposition at hyphal tips and morphogenesis of Streptomyces lividans
}

\author{
Amanda K. Chaplin*, Marloes L.C. Petrus $\dagger$, Giulia Mangiameli†, Michael A. Hough*, Dimitri A. Svistunenko*, Peter Nicholls*, \\ Dennis Claessen $\dagger$, Erik Vijgenboom $\dagger^{+1}$ and Jonathan A.R. Worrall ${ }^{* 1}$ \\ ${ }^{*}$ School of Biological Science, University of Essex, Wivenhoe Park, Colchester, CO4 3SQ, U.K. \\ $†$ Molecular Biotechnology, Institute of Biology, Sylvius Laboratory, Leiden University, PO Box 9505, 2300RA Leiden, The Netherlands
}

\begin{abstract}
Streptomyces lividans displays a distinct dependence on copper to fully initiate morphological development. Evidence has accumulated to implicate the participation of an extracytoplasmic cuproenzyme in morphogenesis. In the present study, we show that GlxA fulfils all criteria to be that cuproenzyme. GlxA is membrane associated and has an active site consisting of a mononuclear copper and a cross-linked Y-C cofactor. The domain organization of the tertiary structure defines GlxA as a new structural member of the mono-copper oxidase family, with copper co-ordination geometry similar to, but spectroscopically distinct from fungal galactose oxidase (Gox). EPR spectroscopy reveals that the oxidation of cupric GlxA generates a protein radical residing on the Y-C cross-link. A variety of canonical Gox substrates (including D-galactose) were tested but none were readily turned over by GlxA. A $g l x A$ null-mutant leads
\end{abstract}

to loss of glycan accumulation at hyphal tips and consequently a drastically changed morphology both on solid substrates and in liquid-grown environments, a scenario similarly observed in the absence of the neighbouring glycan synthase CslA (cellulase synthase-like protein). In addition the glxA mutant has lost the stimulation of development by copper, supporting a model whereby the enzymatic action of GlxA on the glycan is required for development and morphology. From a biotechnology perspective, the open mycelium morphology observed with the glxA mutant in submerged culture has implications for use as an enzyme production host.

Key words: cuproenzyme, development, glycan biotechnology, protein radical, streptomycetes, X-ray structure.

\section{INTRODUCTION}

Streptomycetes are filamentous monoderm soil bacteria that form networks of branching hyphae called mycelia. These organisms display a complex developmental life cycle on solid substrates. Following spore germination, a vegetative mycelium is established that in response to nutrient depletion and other signals initiates both secondary metabolite production and morphological differentiation [1,2]. This leads to the formation of aerial hyphae that will develop into chains of spores. The richness and varied production of secondary metabolites in streptomycetes is coordinated with development and these metabolites have long been a source of interest due to their pharmaceutical properties [1,2]. Streptomycetes also hold promise as a large-scale production host in biotechnology for the heterologous production of proteins and enzymes at high levels for therapeutic, scientific, diagnostic and agricultural purposes [3]. Streptomyces lividans is a preferred choice as an industrial host primarily due to its low level of endogenous extracellular proteolytic activity. As a production host the morphology of the mycelial growth in submerged culture is important as this can play a significant role in its production capacity [4].

Copper $(\mathrm{Cu})$ is an essential redox-active metal ion in living organisms. In S. lividans a distinct dependence on the bioavailability of $\mathrm{Cu}$ in order to fully initiate morphological development has been described [5-7]. In vitro studies have revealed that two extracytoplasmic $\mathrm{Cu}$ metallochaperones, $\mathrm{ECuC}$ (extracytoplasmic $\mathrm{Cu}$ chaperone) and Sco (synthesis of cytochrome c oxidase), facilitate a $\mathrm{Cu}$ trafficking pathway whereby the Sco protein can receive $\mathrm{Cu}$ from $\mathrm{ECuC}$ and deliver it to the $\mathrm{Cu}_{\mathrm{A}}$ site of an $a a_{3}$-type cytochrome $c$ oxidase $(\mathrm{CcO})$. Mutant analysis in S. lividans has revealed that morphological development proceeds in the absence of $\mathrm{ECuC}(\Delta e c u c)$ and $\mathrm{CcO}$ $(\Delta c c o)$, but not in the absence of $\operatorname{Sco}(\Delta s c o)$. Whereas $\Delta e c u c$ and $\Delta s c o$ significantly reduce $\mathrm{CcO}$ activity, these data indicate that morphological development is not linked to impaired $\mathrm{CcO}$ activity [7,8]. Notably the addition of exogenous $\mathrm{Cu}$ to the $\Delta s c o$ mutant rescues development [7]. This implies that a branched $\mathrm{Cu}$ trafficking pathway is prevalent under $\mathrm{Cu}$ homoeostasis conditions in S. lividans, whereby Sco is most certainly required to act as a $\mathrm{Cu}$ chaperone for $\mathrm{CcO}$ and possibly for other extracytoplasmic cupro-proteins/enzymes that trigger formation of aerial hyphae [8].

Analysis of the S. lividans genome has revealed a number of genes that encode putative extracytoplasmic cuproenzymes $[9,10]$. One of these, SLI_3188, has a weak sequence homology with the secreted fungal cuproenzyme galactose oxidase (Gox), with putative ligands to the $\mathrm{Cu}$ ion (two tyrosine and two histidine residues) seemingly conserved (Supplementary Figure S1). Gox houses a catalytic unit, which combines two distinct redox centres; a mononuclear $\mathrm{Cu}$ site capable of one electron redox cycling and a Y-C cofactor, whereby a cysteine residue is cross-linked to one

Abbreviations: $\mathrm{CcO}$, cytochrome c oxidase; CFW, calcofluor white; Gox, galactose oxidase; HRP, horseradish peroxidase; LMCT, ligand-to-metal charge transfer; WT, wild-type.

${ }^{1}$ Correspondence may be addressed to either author (email jworrall@essex.ac.uk or vijgenbo@biology.leidenuniv.nl). 
of the $\mathrm{Cu}$-co-ordinating tyrosine residues and can form a stable protein radical [11-16]. This redox unit enables the two-electron oxidation of a range of D-isomers of primary alcohols (including the C6-hydroxymethyl group of mono- and poly-saccharides) to aldehydes with the reduction of dioxygen to hydrogen peroxide [17]. The physiological function of Gox remains unknown, but the broad substrate specificity inherent in this cuproenzyme suggests that maintaining hydrogen peroxide production in the extracytoplasmic environment may be important for function.

In Streptomyces coelicolor a homologous gene to SLI_3188, annotated as $g l x A$, is required for aerial hyphae development under conditions of osmotic stress [18]. The $g l x A$ gene is the distal gene in an operon with $c s l A$, which encodes a family 2 glycosyltransferase that synthesizes a glycan at hyphal tips $[19,20]$. Orthologues of $g l x A$ and $\operatorname{csl} A$ are present in all streptomycetes and several other actinobacteria with some species having paralogues, e.g. Streptomyces griseus and Streptomyces albus. The genetic locus contains several other genes encoding enzymes implicated in glycan processing, including a $\mathrm{Cu}$ containing lytic polysaccharide mono-oxygenase, suggesting a role of GlxA in polysaccharide modification or synthesis. In the present study, we show that GlxA is a membrane-associated cuproenzyme with a mononuclear $\mathrm{Cu}$ site and a $\mathrm{Y}-\mathrm{C}$ redox cofactor. However, GlxA is distinct from fungal Gox through a unique tertiary structure, atypical spectroscopic properties and a lack of enzymatic activity with a range of 'classical' Gox substrates. As observed in the absence of $c s l A$, we find that a $g l x A$ null-mutant stalls aerial hyphae development on solid media, lacks the hyphal tip glycan and has a dramatically different morphology in liquid-grown cultures. Taken together, our work implies GlxA as the likely candidate mediating $\mathrm{Cu}$-dependent morphogenesis in Streptomyces.

\section{EXPERIMENTAL}

\section{Mycelium washes, fractionation and GIXA detection}

S. lividans 1326 was grown in TSBS (tryptic soy broth supplemented with sucrose) for $18 \mathrm{~h}$ at $30^{\circ} \mathrm{C}$ with shaking at 200 $\mathrm{rpm}$. Mycelium was harvested in $1.5 \mathrm{ml}$ aliquots by centrifugation at $15000 \mathrm{~g}$ for $10 \mathrm{~min}$ and stored at $-80^{\circ} \mathrm{C}$ until required. Mycelium aliquots were first resuspended in $25 \mathrm{mM}$ Tris/ $\mathrm{HCl}$, $\mathrm{pH} 7.5,100 \mathrm{mM} \mathrm{NaCl}$ centrifuged at $25000 \mathrm{~g}$ and the supernatant was kept as the $100 \mathrm{mM} \mathrm{NaCl}$ wash. The pellet was resuspended in buffer containing $1 \mathrm{M} \mathrm{NaCl}$, incubated for $30 \mathrm{~min}$ on ice and centrifuged at $25000 \mathrm{~g}$, which produced the $1 \mathrm{M} \mathrm{NaCl}$ wash. For mycelium extracts the suspended mycelium pellets were sonicated (Bioruptor, 12 cycles: 30-s on, 30-s off). The soluble (S30) and insoluble fractions (P30) were obtained by centrifugation at $30000 \mathrm{~g}$. The supernatant (S30) was subjected to a second centrifugation step at $100000 \mathrm{~g}$ to remove all membrane particles and ribosomes to give the $\mathrm{S} 100$ and $\mathrm{P} 100$ fractions. The pellets (P30 and P100) were resuspended in buffer containing $1 \%$ Triton X-100, incubated on ice for $30 \mathrm{~min}$ and centrifuged again at $30000 \mathrm{~g}$ and $100000 \mathrm{~g}$ respectively to separate Triton soluble (P30-TS, P100-TS) and insoluble (P30-TP, P100-TP) fractions. Fractions obtained were run on SDS/PAGE $(10 \%$ gel) and blotted to Hybond-P membranes for immuno-detection of GlxA and EF-Tu1. The antibodies against GlxA were a kind gift of James W. Whittaker (Oregon Health \& Science University, U.S.A.). The EF-Tu1 antibodies were raised in rabbits against Streptomyces. ramocissimus EF-Tu1 [21]. Incubation with antibodies (GlxA antibodies $10000 \times$ diluted and EF-Tu1 antibodies $5000 \times$ diluted) was carried out for $18 \mathrm{~h}$ at $4{ }^{\circ} \mathrm{C}$ with gentle rocking in PBS with $5 \%$ milk (FrisoLac Extra,
FrieslandCampina). The bound antibodies were detected with GARAP (Sigma) as the secondary antibody and NBT/BCIP (nitro-blue tetrazolium/5-bromo-4-chloro-3-indolyl phosphate) as the substrate. Digital images were taken of the Western blots and total signal intensities were determined with ImageJ [22].

\section{Creation of the $S$. lividans $\Delta \mathrm{cs} \mid \mathrm{A}$ and $\Delta \mathrm{glXA}$ null-mutants, complementation plasmids and analysis of growth morphology}

The $\triangle g l x A$ and $\triangle c s l A$ mutants were prepared and isolated as previously described [7]. In the $\Delta g l x A$ mutant, nucleotides +60 to +1916 relative to the start codon of SLI_3188 were replaced by a $62 \mathrm{nt}$ scar of the loxP recombination site including two XbaI sites. In the $\triangle c s l A$ mutant, nts +79 to +1827 relative to the start of SLI_3187 were replaced. The mutant is consistent with the one described by Xu et al. [20]. Plasmid pGlxA contains the glxA ORF (SLI_3188) and the promoter region $(-545$ to -1$)$ upstream of cslA (SLI_3187). For growth on solid, R5 agar medium [23] was used and supplemented with $\mathrm{Cu}$ as required. Morphology in liquid medium was determined following $24 \mathrm{~h}$ growth with shaking in flasks equipped with coils containing tryptic soya broth (TSB) supplemented with $10 \%$ sucrose and $\mathrm{Cu}$ as indicated. All solid and liquid-grown cultures were incubated at $30^{\circ} \mathrm{C}$. Samples from liquid cultures were analysed by light microscopy with a Zeiss Standard 25 microscope and digital pictures were taken with an AxioCam camera linked to AxioVision software. All spore stocks were obtained from cultures grown on MS (soy flour mannitol medium) agar plates [23] and stored in $20 \%$ glycerol at $-20^{\circ} \mathrm{C}$.

\section{Analysis of glycan production}

Glycan production was determined following $24 \mathrm{~h}$ growth at $30^{\circ} \mathrm{C}$ in eight-well microscopy plates (Lab-TEK II Chambered Coverglass) containing $500 \mu \mathrm{l}$ of mNMMP (minimal NMMP medium with mannitol) medium [24]. Mycelium was stained by adding $50 \mu \mathrm{l}$ of calcofluor white (CFW) solution (Remel Bactidrop) to each well. After $5 \mathrm{~min}$, but within $20 \mathrm{~min}$, stained mycelium was analysed with a laser-scanning confocal microscope (Zeiss LSM5 Exciter/Axio Observer) by excitation with a $405 \mathrm{~nm}$ laser, a 405/488 $\mathrm{nm}$ beamsplitter and a bandpass emission filter of $420-480 \mathrm{~nm}$. Images were adjusted for brightness and contrast using Image J [22].

\section{Cloning, overexpression and purification of GIXA}

The $g l x A$ gene with 200 flanking nt at the 5'- and $3^{\prime}$-ends was amplified from $S$. lividans 1326 and cloned into a pUC19 vector. This construct was used to create an $\mathrm{N}$-terminal deleted $g l x A$ gene for overexpression in Escherichia coli by amplifying the $g l x A \mathrm{nt}$ sequence that starts coding for amino acid 35 and restricted using the NdeI and BamHI sites of a pET28a vector (Novagen) to create an $\mathrm{N}$-terminal $\mathrm{His}_{6}$-tagged $g l x A$ construct. The overexpression and purification of recombinant GlxA are described in Supporting Information.

\section{Preparation of GIxA samples and UV-visible spectroscopy}

GlxA concentration was determined by UV-visible spectroscopy (Varian Cary 50 UV-visible spectrophotometer) using an absorption coefficient $(\varepsilon)$ at $280 \mathrm{~nm}$ of $78730 \mathrm{M}^{-1} \cdot \mathrm{cm}^{-1}$. The various forms of GlxA were prepared through the addition of $\sim 5$-fold excess of $\left[\mathrm{Fe}(\mathrm{CN})_{6}\right]^{3-}$ (Sigma), $\left[\mathrm{Ir}(\mathrm{Cl})_{6}\right]^{3-}$ (Acros) or $\mathrm{Na}_{2} \mathrm{~S}_{2} \mathrm{O}_{4}$ (Sigma) followed by removal via a PD-10 column (GE Healthcare). 
Table 1 X-ray data processing and refinement parameters

The GIXA crystal structure was in space group P2 ${ }_{1}$ with unit cell parameters 50.4, 126.6, 107.6 $\AA, 90,91.1,90^{\circ}$. Values in parentheses refer to the outermost resolution shell (1.80-1.77 $\AA$ ).

\begin{tabular}{ll}
\hline Resolution $(\AA)$ & $53.3-1.77$ \\
\hline Unique reflections & $130533(21898)$ \\
Completeness $(\%)$ & $99.7(99.6)$ \\
Redundancy & $3.6(3.4)$ \\
$R_{\text {merge }}$ & $0.064(0.633)$ \\
Mn(I/sd) & $10.5(2.0)$ \\
Wilson B factor $\left(\AA^{2}\right)$ & 20.5 \\
$R_{\text {cryst }}$ & 0.188 \\
$R_{\text {free }}$ & 0.228 \\
RMSD bond lengths $(\AA)$ & 0.011 \\
RMSD bond angles ( $\left.{ }^{\circ}\right)$ & 1.41 \\
ESU based on ML $(\AA)$ & 0.096 \\
Ramachandran favoured $(\%)$ & 95.9 \\
PDB accession code & 4 unm \\
\end{tabular}

\section{Crystallization and structure determination}

An ARI-Gryphon 96-well crystallization robot was used to screen crystallization conditions for GlxA. A crystal hit was discovered in $0.1 \mathrm{M}$ sodium acetate, $\mathrm{pH}$ 4.6, $15 \%$ PEG 20000 (PEG suite, Qiagen). Scaling-up and optimization of GlxA crystals from the initial hit was carried out in 24-well VDX plates (Molecular Dimensions) using the hanging drop vapour diffusion method at $20^{\circ} \mathrm{C}$. Equal volumes of GlxA solution at a concentration of $15 \mathrm{mg} \cdot \mathrm{ml}^{-1}$ and reservoir solution containing $0.1 \mathrm{M}$ sodium acetate, $\mathrm{pH}$ 4.0, $20 \%$ PEG 20000 were mixed. Crystals suitable for diffraction studies grew within 1 week. A single crystal was transferred to a cryoprotectant solution containing the respective reservoir solution and $20 \%$ glycerol prior to flash-cooling by plunging into liquid nitrogen. Crystallographic data were measured at the beamline I03, Diamond Light Source, using an X-ray wavelength of $0.979 \AA(1 \AA=0.1 \mathrm{~nm})$ and a Pilatus $6 \mathrm{M}-\mathrm{F}$ detector (Dectris). Details of structure determination are given in Supporting Information. Co-ordinates and structure factors were deposited in the RCSB Protein Data Bank with accession number 4unm. A summary of data and refinement statistics and the quality indicators for the structure are given in Table 1. CAVER analyst 1.0 was used for the identification of tunnels [25]. Chain B of GlXA was analysed using the tunnel computation tool within CAVER with a starting point defined using the first co-ordination sphere $\mathrm{Cu}$-binding residues $\left(\mathrm{Tyr}^{289}, \mathrm{Tyr}^{501}, \mathrm{His}^{502}, \mathrm{His}^{589}\right)$. Default settings for tunnel analysis were used, including the minimum probe radius set to $0.9 \AA$.

\section{EPR spectroscopy}

GlxA samples (85-120 $\mu \mathrm{M})$ for EPR were prepared in duplicate in a mixed buffer system consisting of $10 \mathrm{mM}$ each of Tris, potassium acetate, MES, MOPS and $200 \mathrm{mM} \mathrm{KCl}$ with the $\mathrm{pH}$ adjusted to 7.0. Wilmad SQ EPR tubes (Wilmad Glass) were filled with the GlxA solutions and frozen in methanol kept on dry ice. The tubes were then transferred to liquid nitrogen. All EPR spectra were measured on a Bruker EMX EPR spectrometer (X band). A spherical high-quality Bruker resonator ER 4122 SP 9703 and an Oxford Instruments liquid helium system were used to measure the low-temperature EPR spectra. Digitizing of a published EPR spectrum was performed using Un-Scan-It, v.6, Silk Scientific.

\section{Activity assays}

A variety of putative substrates for GlxA were assayed. These included the following monosaccharides; D-galactose, D-glucose, D-sucrose, D-fructose $N$-acetyl-D-glucosamine and D-glucuronic acid; the disaccharides, D-lactose and D-cellobiose; and the aldehydes and primary alcohols glycolaldehyde, glyoxal and glycerol (all purchased from Sigma). Catalytic turnover was measured using a coupled assay, whereby the production of $\mathrm{H}_{2} \mathrm{O}_{2}$ by GlxA was detected by the presence of horseradish peroxidase (HRP; Sigma) and the subsequent oxidation of guaiacol (Sigma). Samples were prepared in $3 \mathrm{ml}$ quartz cuvettes containing $0.1 \mathrm{M}$ sodium phosphate, $\mathrm{pH} 7.4,1 \mathrm{mM}$ guaiacol, $1 \mu \mathrm{l}$ of HRP $\left(10 \mathrm{mg} \cdot \mathrm{ml}^{-1}\right), 20-30 \mu \mathrm{M}$ GlxA and various concentrations of the individual substrate. The oxidation of guaiacol was monitored at $470 \mathrm{~nm}$ using a Hewlett-Packard 8453 diode-array spectrophotometer scanning between 190 and $1100 \mathrm{~nm}$ and thermostatted at $25^{\circ} \mathrm{C}$. Plots of turnover rate $\left(k, \mathrm{~s}^{-1}\right)$ compared with substrate concentration were constructed, whereby $k$ was calculated from $\left.\left(\left(\Delta A_{470} / \varepsilon_{\mathrm{gc}}\right) / t\right) /[\mathrm{GlxA}]\right)$ where $\Delta A_{470}$ is the absorbance change at $470 \mathrm{~nm}$ upon guaiacol oxidation, $\varepsilon_{\mathrm{gc}}$ is the absorption coefficient of the guaiacol oxidation product taken as $5.57 \mathrm{mM}^{-1} \cdot \mathrm{cm}^{-1}, t$ is the time in seconds and [GlxA] is the total millimolar concentration of GlxA in the assay.

\section{RESULTS}

\section{GIXA is associated with the membrane}

The distribution of GlxA in the soluble and insoluble fractions of liquid grown $S$. lividans mycelium was first determined. Upon thorough mycelium sonication followed by centrifugation steps, a small fraction of GlxA was detected in the soluble protein fraction (S100) with the majority remaining in the pellet fraction (P30 and P100; Figures 1A and 1B). In contrast, the majority of the cytoplasmic marker EF-Tu1 is detected in the soluble fraction (S100; Figures 1A and 1B). Resuspension of the insoluble pellet fraction in buffer containing $1 \%$ Triton X-100 solubilizes a significant amount of GlxA (Figures $1 \mathrm{~A}$ and $1 \mathrm{~B}$ ), indicating that GlxA is not covalently bound to the membrane. However, salt washes did not remove GlxA from intact mycelium (Figure 1C), but small amounts of EF-Tu1 were detected probably due to some lysis. These data show that GlxA is not a peripheral membrane protein but is firmly associated with the membrane, although not via a covalent bond. These results are in agreement with in silico analysis, which predicts residues $1-11$ of GlxA to be an Nterminal signal peptide [26], with a weak signal peptidase cleavage site between residues $11-12$, followed by a transmembrane helix (residues 12-32), which could function as the membrane anchor $[27,28]$.

\section{The tertiary structure of GIXA consists of three distinct domains}

To elucidate the molecular features of GlxA, an N-terminal truncated expression construct $(\Delta 1-34)$ for overexpression in E. coli was generated, which yielded $45 \mathrm{mg} \cdot \mathrm{1}^{-1}$ of purified GlxA. The crystal structure of GlxA was determined to $1.77 \AA$ resolution and contains two protein molecules in the crystallographic asymmetric unit. Molecule A (residues 38-645) exhibits significantly lower B-factors and higher quality electron density than molecule $\mathrm{B}$, but contains a disordered region between residues 198 and 206 (not visible in the electron density), whereas the main chain for molecule B was complete between residues 37 and 645. The overall structure of GlxA (Figure 2) consists of three predominately $\beta$-sheet domains. Preceding the first domain 


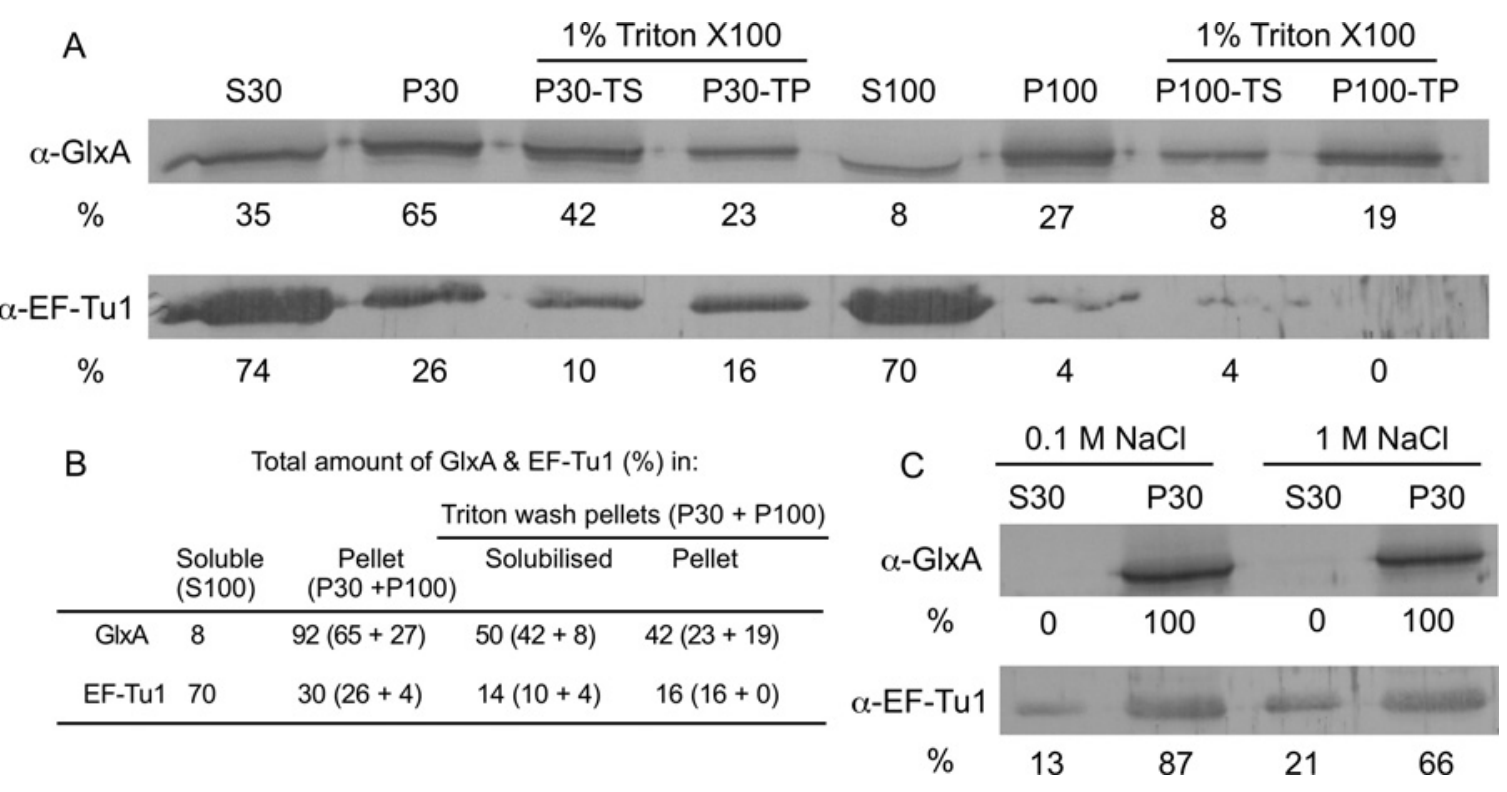

\section{Figure 1 Location of GIXA in the mycelium determined by Western blotting}

(A) Detection of GlxA and EF-Tu1 in the various mycelium fractions. Samples were prepared as described in 'Experimental'. The band intensities are expressed as percentages relative to the total amount of GIXA or EF-Tu1 in the S30 plus P30 fraction, the intensity of which was set at 100\%. (B) The Table presents the total soluble (S100), 1\% triton soluble (P30-TS + P100-TS) and insoluble fraction (P30-TP + P100-TP) of GlxA and EF-Tu1 expressed as a percentage according to (A). (C) NaCl washes of the intact mycelium. The signals detected in the S30 plus P30 samples of the $100 \mathrm{mM} \mathrm{NaCl}$ wash were set at $100 \%$.

are two short $\mathrm{N}$-terminal $\alpha$-helices ( $\alpha 1$ and $\alpha 2$ ), with the $\alpha 1$ helix orientated approximately perpendicular to the $\alpha 2$ helix. Domain 1 of GlxA consists of seven Kelch motifs (blades) arranged in a $\beta$ propeller tertiary structure (Figure 2). Each blade of the propeller consists of four anti-parallel $\beta$-sheets $(a, b, c, d)$ with each sheet connected through variable length loops (Figure 2). Blades 1 and 2 each possess structural inserts in the loops connecting sheet $b$ to sheet $c$. In blade 1 a short platform-like $\alpha$-helix $(\alpha 3)$ is inserted which has an overall negative charge, whereas in blade 2 , sheets $b$ and $c$ are connected via domain 2 (Figure 2). Domain 2 consists of $10 \beta$-sheets and 1 short $\alpha$-helix $(\alpha 4)$. According to the CATH database [29] the domain 2-fold is classified as mainly $\beta$, with a $\beta$ barrel architecture formed by sheets $1,3,4,8,9$ and 10 (Figure 2) and a representative domain topology with thrombin, subunit $\mathrm{H}$. Protruding out from the core $\beta$-barrel is a long $\beta$-hairpin loop (residues 194-210) consisting of $\beta$-sheets 6 and 7 (Figure 2). In molecule $\mathrm{A}$, this $\beta$-hairpin loop is disordered and not built into the model, whereas this is not the case for molecule B due to stabilizing polar interactions with molecule $A$ residues from a symmetry related GlxA molecule in the unit cell (Supplementary Figure S2). Domain 2 is positioned in the overall tertiary structure such that the $\beta$-barrel core lies across the top of approximately half of domain 1 with the $\beta$-hairpin loop extending into the solvent (Figure 2). Further analysis of domain 2 using PDBeFold identifies no structures deposited in the PDB with a high structural homology (the highest Q-score obtained was only 0.14). Thus the fold of domain 2 in GlxA is relatively novel compared with known structures. The third domain starts immediately after the seventh Kelch motif and is located at the bottom of domain 1. Domain 3 consists of eight $\beta$-sheets and one short $\alpha$-helix forming a $\beta$ sandwich fold with a high structural homology to domain 3 of Gox (1.18 $\AA$ RMSD from superimposition). A loop (residues $583-590$ in GlxA) connecting $\beta$-sheets 3 and 4 of domain 3 penetrates upwards into domain 1 along the 7-fold symmetry axis and provides one of the ligands $\left(\mathrm{His}^{589}\right.$ ) to the $\mathrm{Cu}$ ion of
GlxA (see below). The charge distribution of GlxA (pI 8.2) is displayed through electrostatic surface representations (Figure 2) and indicates a rather disperse distribution of charge across the whole molecular surface.

\section{Architecture and spectroscopic properties of the Cu site in GIXA}

A well-defined $16 \sigma$ peak in the $\sigma$-weighted $\mathrm{F}_{\mathrm{o}}-\mathrm{F}_{\mathrm{c}}$ difference map was present in each GlxA chain in the asymmetric unit, into which a $\mathrm{Cu}$ ion was modelled. The $\mathrm{Cu}$ is co-ordinated in a square pyramidal geometry with the $\mathrm{OH}$ of $\mathrm{Tyr}^{501}$ acting as the axial ligand and the equatorial coordination positions occupied by the $\mathrm{OH}$ of $\mathrm{Tyr}^{289}$, the $\mathrm{N} \varepsilon 2$ of $\mathrm{His}^{502}$ and $\mathrm{His}^{589}$ and $\mathrm{a}_{2} \mathrm{O}$ molecule (Figure 3A). Bond lengths to the $\mathrm{Cu}$ ion are reported in Table 2 together with a comparison to those in Gox. Unbroken electron density is observed between the side chain $\mathrm{S} \gamma$ atom of Cys ${ }^{121}$ and the $\mathrm{C} \varepsilon 1$ ring atom of $\mathrm{Tyr}^{289}$, providing clear evidence that a cross-linked Y-C cofactor is formed in GlxA, with a bond length of $1.9 \AA$ (2.0 $\AA$ in chain B; Figure $3 \mathrm{~A})$. Adjacent to $\mathrm{Tyr}^{289}$ is $\operatorname{Trp}^{288}$, which has its side chain indole ring orientated such that the benzene ring is $\pi-\pi$ stacking with the phenoxyl ring of $\mathrm{Tyr}^{289}$ with $C^{\text {Tyr }}-C^{\text {Trp }}$ distances between $\sim 3.5$ and $4 \AA$ (Figure $3 \mathrm{~A}$ ).

The UV-Vis absorbance spectrum of the blue-grey $\mathrm{Cu}$ (II)-GlxA has a broad, low intensity band in the visible region $\left(\lambda_{\max } \sim\right.$ $577 \mathrm{~nm})$ and two distinct shoulders $\left(\lambda_{\max } \sim 362\right.$ and $320 \mathrm{~nm}$; Figure 3B). No change to these absorption features in the $\mathrm{pH}$ range 4-8 was observed, with the addition of $\mathrm{Cu}(\mathrm{II})$ ions resulting in only a small absorbance increase in $\lambda_{\max } \sim 577 \mathrm{~nm}$ over a 12-h period (Figure $3 \mathrm{~B}$ ). In contrast, the $\mathrm{Cu}(\mathrm{II})$-Gox absorption spectrum has weak intensity bands at $\lambda_{\max } 441 \mathrm{~nm}$ attributed to phenolate (Tyr) to $\mathrm{Cu}$ (II) ligand-to-metal charge transfer (LMCT) and at $\lambda_{\max } 630 \mathrm{~nm}$ arising from mixed $\mathrm{Cu}$ ligand field transition and LMCT $[11,14]$. Addition of the reductant $\mathrm{Na}_{2} \mathrm{~S}_{2} \mathrm{O}_{4}$ bleaches all absorption features (Figure $3 \mathrm{~B}$ ) with removal of $\mathrm{Na}_{2} \mathrm{~S}_{2} \mathrm{O}_{4}$ and 


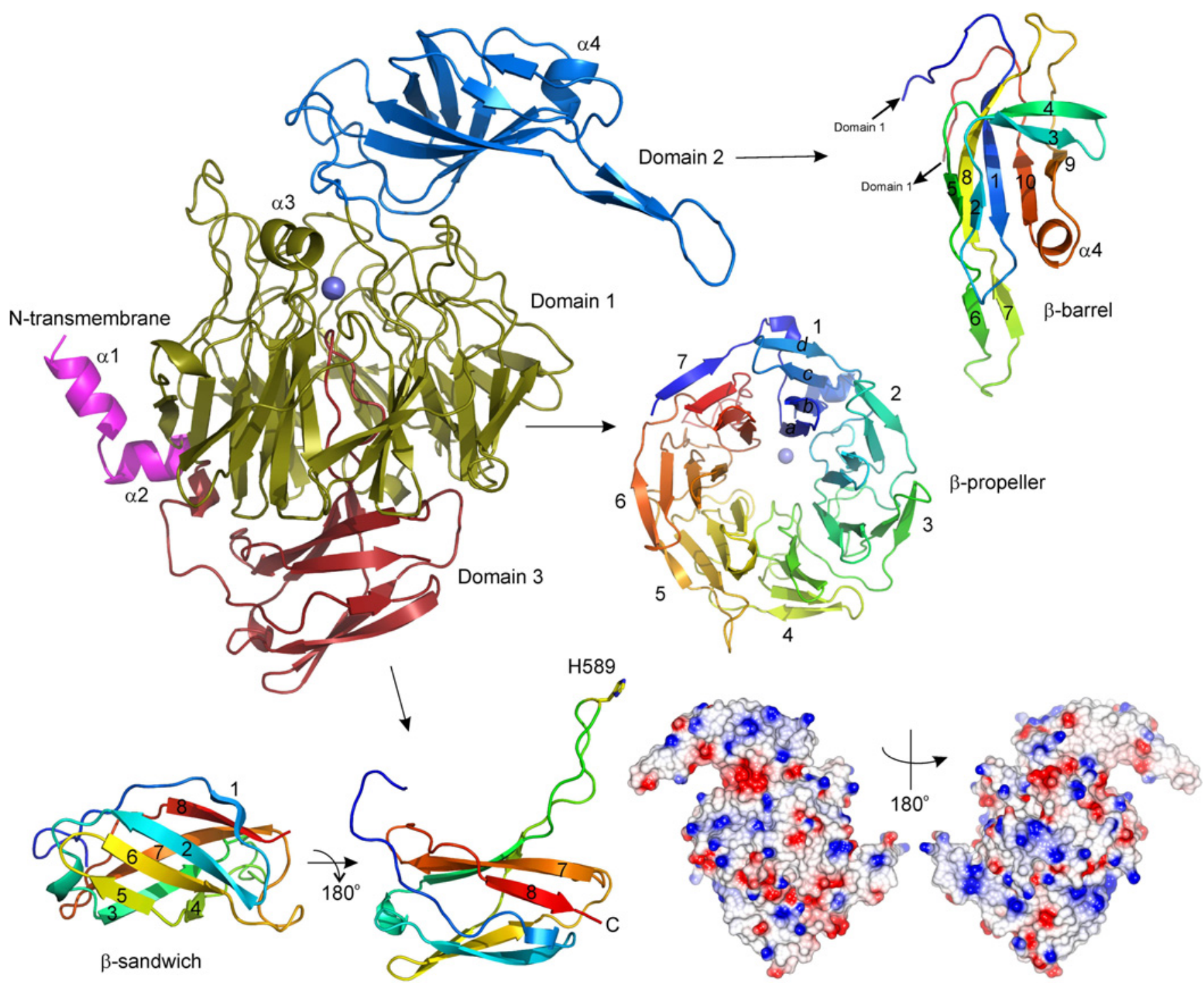

Figure 2 X-ray crystal structure of $S$. lividans GIXA

The three domains forming the tertiary structure are indicated and coloured gold (domain 1), blue (domain 2) and red (domain 3). The four short $\alpha$-helices present in the structure are labelled. Each domain is shown individually and in more detail as discussed in the main text. The Cu atom is represented as a sphere and electrostatic surface representations in two GlxA orientations are shown. Images were prepared in PyMol and CCP4MG [48].

subsequent exposure to air resulting in the return of the $\mathrm{Cu}$ (II)GlxA spectrum. This infers that the $\mathrm{Cu}$ is redox active and can cycle between the $\mathrm{Cu}$ (II) and $\mathrm{Cu}(\mathrm{I})$ states.

The EPR spectrum of $\mathrm{Cu}(\mathrm{II})-\mathrm{GlxA}$ at $\mathrm{pH} 7$ displays features consistent with an axial $\mathrm{Cu}(\mathrm{II}) \mathrm{g}$-tensor, $\mathrm{g}_{\|}=2.182$ and $\mathrm{g}_{\perp}=2.05$ (Figure 3C). The strong line on the right from the indicated $g_{\perp}$ region is not a principal g-factor component but an 'overshoot' line [30] occurring for specific orientations of the $\mathrm{Cu}$ (II) complexes, subject to particular relationships between the values of $g_{\|}, g_{\perp}$ and the anisotropic components of the hyperfine interaction of the electron spin $(S=1 / 2)$ with the $C u$ nuclear $\operatorname{spin}(I=3 / 2), A_{\|}$and $A_{\perp}$. The EPR spectrum line shape is similar to that of $\mathrm{Cu}$ (II)-Gox [31], which also has an axial $\mathrm{Cu}$ (II) g-tensor [32-34]. However, the GlxA spectrum exhibits a more distinctly resolved overshoot line, likely to be a consequence of a smaller $\mathrm{g}_{\|}$(2.182 as compared with 2.21-2.23 in Gox). The $\mathrm{g}_{\perp}$ component displays a hyperfine interaction of the electron spin with the $\mathrm{Cu}$ (II) ligands, which for
GlxA gives seven lines with relative intensities of 1:4:8:10:8:4:1 (Figure 3C, inset) compared with five lines in Gox [33,34].

\section{Substrate access sites and binding pocket}

Due to the buried nature of the $\mathrm{Cu}$ site, it is likely that a substrate would have to gain access via a tunnel. CAVER was used to identify tunnels in the GlxA structure [25,35], defined as void pathways leading from a cavity inside GlxA, i.e. the $\mathrm{Cu}$ site, that transverse to the protein surface. Three tunnels of different lengths were identified (Figure 4A). The entrance to tunnel I lies between the $\alpha 3$ helix on the loop connecting sheets $a$ and $b$ in blade 1 and a well-ordered loop in blade 7 of domain 1 (Figures $4 \mathrm{~A}$ and $4 \mathrm{~B}$ ). The tunnel has an opening of $\sim 8 \times 8 \AA$, is void of $\mathrm{H}_{2} \mathrm{O}$ molecules and leads directly to the equatorially $\mathrm{Cu}$ co-ordinating $\mathrm{H}_{2} \mathrm{O}$ molecule, with a distance to the surface 

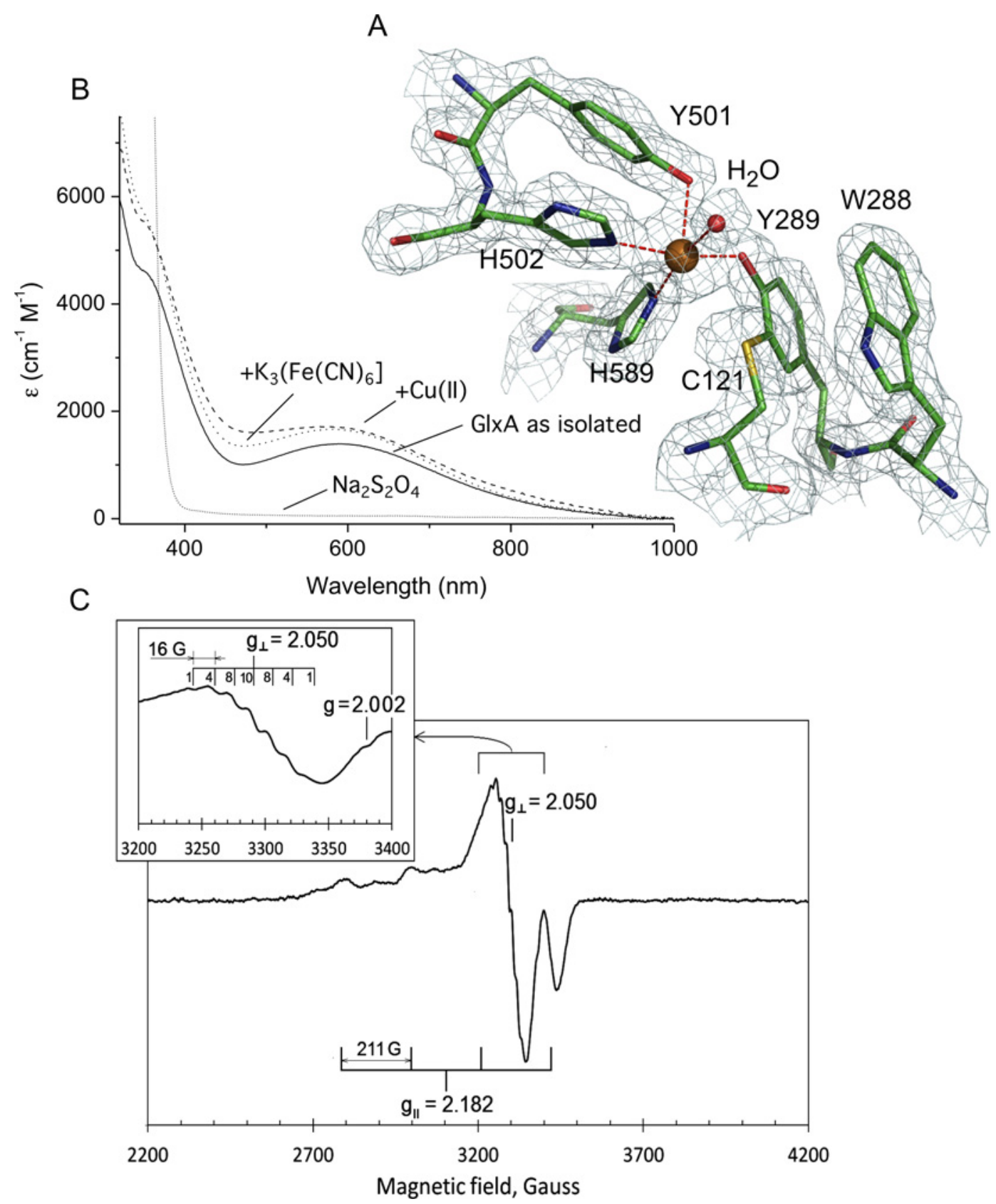

\section{Figure 3 Structure and spectroscopy of the GIXA Cu site}

(A) $2 \mathrm{~F}_{0}-\mathrm{F}_{\mathrm{c}}$ electron-density map contoured at $2 \sigma$ of the $\mathrm{Cu}$ site in GIXA. The $\mathrm{Cu}$ ion is represented as a brown sphere with the ligands to the Cu ion depicted as sticks and the equatorially coordinating $\mathrm{H}_{2} \mathrm{O}$ molecule as a red sphere. (B) Absorbance spectra at pH 7 and $20^{\circ} \mathrm{C}$ of purified $\mathrm{GIXA}(80 \mu \mathrm{M})$. (C) $\mathrm{Cu}(\mathrm{II})$-GIXA EPR spectrum $(85 \mu \mathrm{M})$ at pH 7 . The g of the electron $\operatorname{spin}(S=1 / 2)$ with the $I=3 / 2$ Cu nuclear spin, four components $1: 1: 1: 1$ separated by $A_{Z}{ }^{C u}=211 \mathrm{G}$. Inset: the $g_{\perp}$ region of the spectrum, which displays unpaired electron $(S=1 / 2)$ hyperfine interaction with the $\mathrm{Cu}(\mathrm{II})$ ligands, seven components of relative intensities 1:4:8:10:8:4:1 separated by $16 \mathrm{G}$. Instrumental conditions: temperature $10 \mathrm{~K}$, microwave frequency $v_{\mathrm{Mw}}=9.47$ $\mathrm{GHz}$, microwave power $P=3.18 \mathrm{~mW}$, modulation frequency $\nu_{\mathrm{M}}=100 \mathrm{kHz}$, modulation amplitude $A_{\mathrm{M}}=5 \mathrm{G}$, time constant $\tau=82 \mathrm{~ms}$, scan rate $V=22.6 \mathrm{G} \cdot \mathrm{s}^{-1}$, number of scans per spectrum $\mathrm{NS}=1$.

of $\sim 10 \AA$. The dimensions of the surface opening are such that pyranose carbohydrates, e.g. D-glucose or D-galactose and also C2 substituted pyranose carbohydrates such as $N$-acetyl-glucosamine would be able to pass. The opening of tunnel II is located beneath the $\beta$-hairpin loop of domain 2 (Figure 4B), with the dimensions of the opening, $\sim 9 \times 7 \AA$. A network of six $\mathrm{H}$-bonded $\mathrm{H}_{2} \mathrm{O}$ molecules are present in the tunnel that thread down to the $\mathrm{Cu}$ coordinating $\mathrm{H}_{2} \mathrm{O}$ molecule, with a distance to the surface of $\sim 25 \AA$. Tunnel III spans a large section of domain 1, starting from below the equatorial histidine ligands and leading to a surface entrance formed by residues $\mathrm{Ala}^{128}, \mathrm{Gly}^{298}, \mathrm{Pro}^{322}$ and $\mathrm{Ser}^{622}$. The tunnel is lined with a continuous network of well-ordered $\mathrm{H}_{2} \mathrm{O}$ molecules (13 in total) and has a distance from the $\mathrm{Cu}$ ion to the surface of $\sim 70 \AA$. A putative substrate pocket is identified above the axial $\mathrm{Tyr}^{501}$ ligand and is directly accessible from either tunnel I or II (Figure 4C). The pocket is occupied by several $\mathrm{H}_{2} \mathrm{O}$ molecules, all of which are well ordered and form an extensive $\mathrm{H}$-bond network, which also includes an $\mathrm{H}$-bond interaction with the $\mathrm{Tyr}^{501}$ ligand 
A

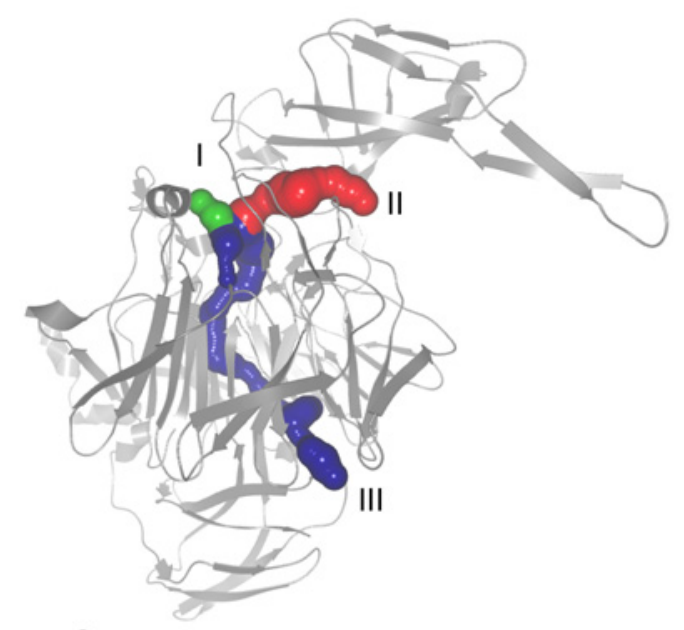

C

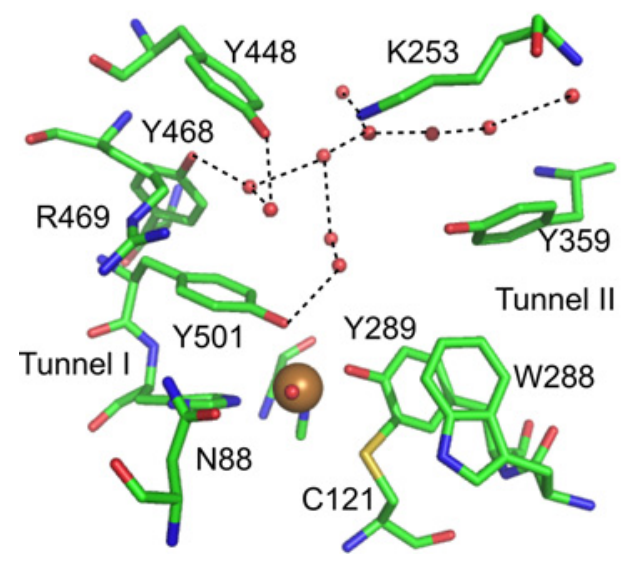

B

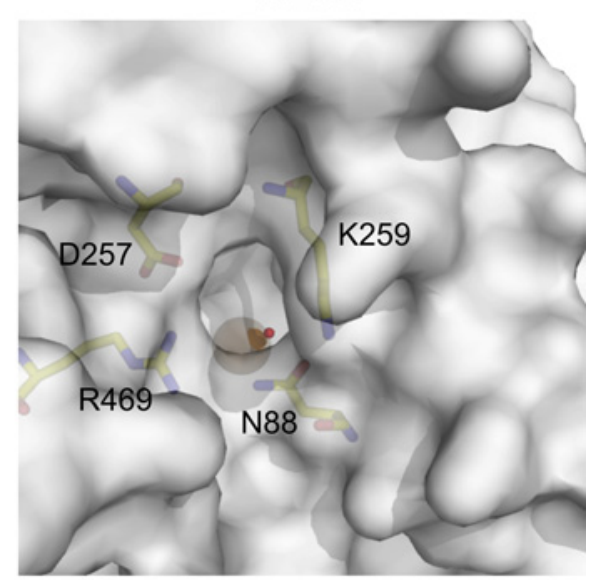

Tunnel II

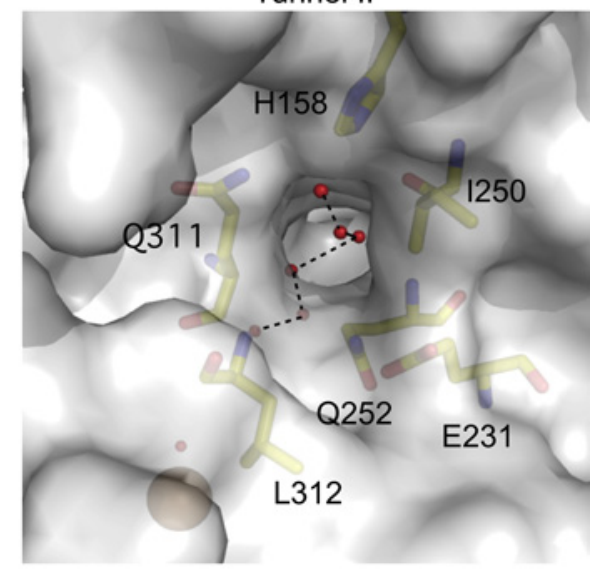

Figure 4 Substrate access channels and binding pocket

(A) Location of the three surface-to-Cu site tunnels (I, II, III) in S. lividans GIXA identified using the program CAVER [25]. (B) Partial transparent surface views of the openings to tunnels I and II. The amino acids forming the openings are labelled and shown as sticks, $\mathrm{H}_{2} \mathrm{O}$ molecules are depicted as red spheres and the $\mathrm{Cu}$ ion as a brown sphere. In tunnel I the equatorially $\mathrm{Cu}$ co-ordinated $\mathrm{H}_{2} \mathrm{O}$ molecule is visible and tunnel II illustrates the $\mathrm{H}$-bonded $\mathrm{H}_{2} \mathrm{O}$ network leading from the surface to the Cu site. (C) The putative substrate-binding pocket in GlXA.

Table 2 Bond lengths of the Cu sites (monomer A and B) in S. livdians GIXA and F. graminearum Gox (PDB 1gof) [13]

\begin{tabular}{|c|c|c|c|}
\hline \multicolumn{2}{|l|}{$\mathrm{G} \mid \mathrm{XA}(\mathrm{A} / \mathrm{B})$} & \multicolumn{2}{|l|}{ Gox } \\
\hline $\mathrm{Cu}-\mathrm{Tyr}^{289} \mathrm{OH}$ & $1.84 / 1.97 \AA$ & Cu-Tyr ${ }^{272} \mathrm{OH}$ & $1.93 \AA$ \\
\hline Cu-Tyr ${ }^{501} \mathrm{OH}$ & $2.24 / 2.14 \AA$ & Cu-Tyr ${ }^{495} \mathrm{OH}$ & $2.69 \AA$ \\
\hline $\mathrm{Cu}-\mathrm{His}^{502} \mathrm{~N} \varepsilon 2$ & $2.13 / 2.18 \AA$ & $\mathrm{Cu}-\mathrm{His}^{496} \mathrm{~N} \varepsilon 2$ & $2.11 \AA$ \\
\hline $\mathrm{Cu}-\mathrm{His}^{589} \mathrm{~N} \varepsilon 2$ & $2.18 / 2.23 \AA$ & $\mathrm{Cu}-\mathrm{His}^{581} \mathrm{~N} \varepsilon 2$ & $2.14 \AA$ \\
\hline $\mathrm{Cu}-\mathrm{H}_{2} \mathrm{O}$ & $2.44 / 2.50 \AA$ & ${ }^{\star}$ Cu-acetate & *2.26 \\
\hline Tyr $^{289}-$ Cys $^{121}$ & $1.92 / 1.97 \AA$ & Tyr-Cys & $1.83 \AA$ \\
\hline
\end{tabular}

*1gof crystallized in acetate buffer, pH4.5, (GIXA acetate buffer, pH 4.0), with an acetate molecule found in place of the $\mathrm{H}_{2} \mathrm{O}$ molecule in GIXA. In a Gox structure (1gog) [13] crystallized in the absence of acetate buffer at pH $7.0 \mathrm{a} \mathrm{H}_{2} \mathrm{O}$ molecule is found with a bond length to the $\mathrm{Cu}$ ion of $2.81 \AA$.

(Figure 4C). The pocket is completely polar and is formed from the side chains of six amino acids (Figure 4C). Side-chain H-bonding interactions with the $\mathrm{H}_{2} \mathrm{O}$ network in the pocket are apparent for some of the residues (Figure 4C), with $\mathrm{Asn}^{88}$ and $\mathrm{Arg}^{469}$, part of the entrance to tunnel I (Figure 4B), also capable of H-bond interactions.

\section{The $Y-C$ cross-link is redox active}

The Y-C cross-link identified in the GlxA X-ray structure is redox active in Gox. Addition of the oxidants, $\left[\mathrm{Fe}(\mathrm{CN})_{6}\right]^{3-}$ or $\left[\operatorname{Ir}(\mathrm{Cl})_{6}\right]^{3-}$, to $\mathrm{Cu}(\mathrm{II})-\mathrm{GlxA}$ does not perturb the absorbance spectrum (Figure 3B) in contrast with Gox where formation of the fully oxidized $\mathrm{Cu}$ (II)-Tyr-Cys• form leads to distinct spectral features. However, a change in the EPR spectrum of GlxA following addition of $\left[\mathrm{Fe}(\mathrm{CN})_{6}\right]^{3-}$ is observed, with a three-line free radical EPR spectrum detected (Figure 5). The spectrum is very similar to the spectrum of the radical on the Y-C cross-link reported for Gox [12], glyoxal oxidase [36] and SCO2837p from S. coelicolor [37] (Figure 5). This observation taken together with the result of $\mathrm{Na}_{2} \mathrm{~S}_{2} \mathrm{O}_{4}$ reduction indicates that the active site of GlxA can access three oxidation states [reduced $(\mathrm{Cu}(\mathrm{I})]$, semi-reduced $[\mathrm{Cu}(\mathrm{II})]$ and fully oxidized [Cu(II)-Tyr-Cys•)] as is the case for Gox and should therefore be able to catalyse the two-electron oxidation of a substrate. 


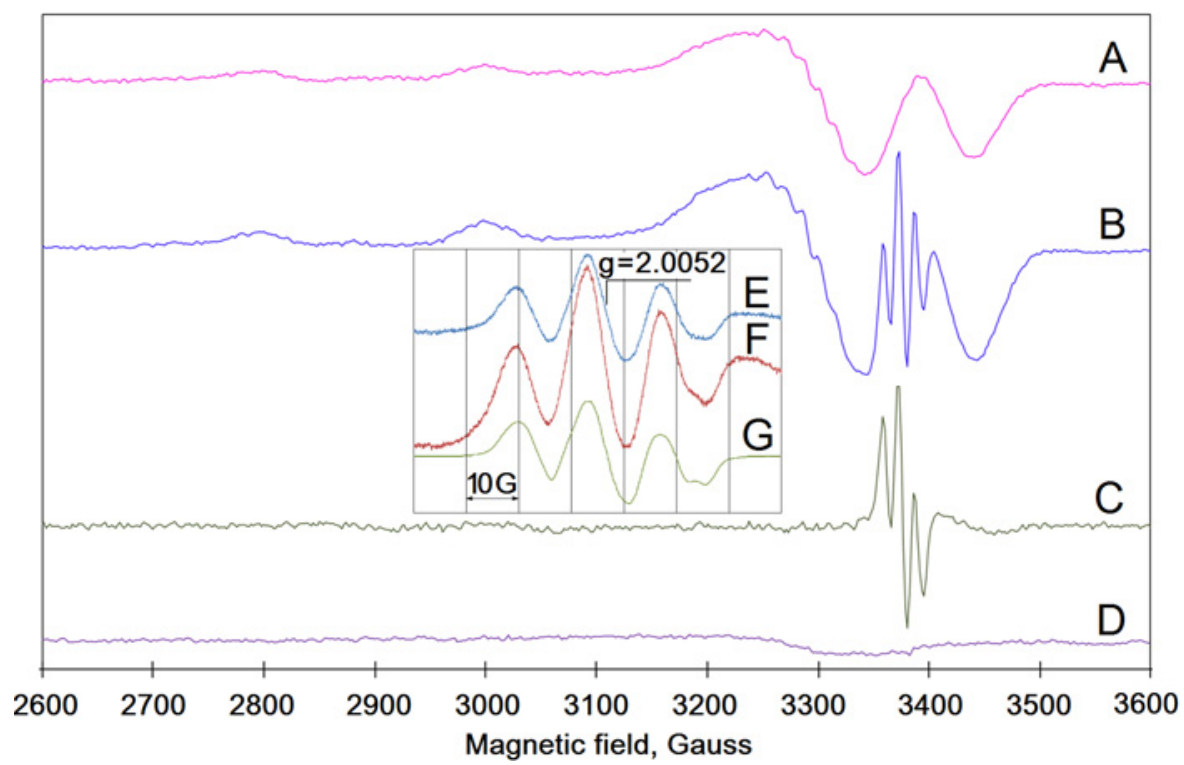

Figure 5 EPR spectra of Y-C radicals

(A) As isolated $\mathrm{GlXA},(\mathbf{B})$ after $\left[\mathrm{Fe}(\mathrm{CN})_{6}\right]^{3-}$ treatment, (C) difference spectrum $(\mathrm{B}-1.46 \times \mathrm{A})$ showing the free radical EPR signal in its pure form (the coefficient 1.46 was found empirically to minimize input of other EPR signals to the difference spectrum), (D) as in $(\mathbf{A})$, but after $\mathrm{Na}_{2} \mathrm{~S}_{2} \mathrm{O}_{4}$ treatment. Inset: the same sample as the one used to detect spectrum (B), was used to measure the free radical EPR signal in greater detail using two different microwave power levels, $50 \mu \mathrm{W}(\mathbf{E})$ and $3.18 \mathrm{~mW}(\mathbf{F})$. (G) The EPR spectrum of the radical in SC02837p from S. coelicolor [37]. All spectra were recorded at $40 \mathrm{~K}$ using $120 \mu \mathrm{M} \mathrm{GIXA}$ at pH 7, with the instrumental conditions for $(\mathbf{A}, \mathbf{B}$ and $\mathbf{D})$ the same as in Figure $3(\mathrm{C})$ and for spectra $(\mathbf{E})$ and $(\mathbf{F})$ as follows: modulation amplitude $A_{\mathrm{M}}=3 \mathrm{G}$, scan rate $V=0.596 \mathrm{G} \mathrm{s}^{-1}$. Spectrum vii is a digitized image from [37], aligned with the GlxA spectrum on the basis of $\mathrm{g}$-factors, therefore the magnetic field axis is not indicated.

\section{Enzymatic activity of GlxA}

A number of compounds were assayed for activity with GlxA using a coupled peroxidase assay (see 'Experimental'), with only four showing significant oxidase activity. Figure 6(A) reveals that aerobic oxidation rates for D-galactose, D-glucose and glycerol, follow a linear substrate relationship for the concentrations used. From the slopes of these plots, second order rate constants $\left(k_{\text {red }}\right)$ were determined for D-galactose, $8.4 \times 10^{-3} \mathrm{M}^{-1} \cdot \mathrm{s}^{-1}$; for D-glucose, $8.8 \times 10^{-3} \mathrm{M}^{-1} \cdot \mathrm{s}^{-1}$; and for glycerol, $1.7 \times 10^{-2}$ $\mathrm{M}^{-1} \cdot \mathrm{s}^{-1}$. No activity was observed for the $\mathrm{C} 2$ position modified monosaccharide $N$-acetyl-D-glucosamine or for fructose, a fivemembered ring monosaccharide. Likewise D-glucuronic acid gave no activity, illustrating that a $\mathrm{C} 1$ glycosidic (aldehyde) group is not effective as a reductant when the C6 group is modified ( $\mathrm{COOH}$ compared with $\mathrm{CH}_{2} \mathrm{OH}$ ). Furthermore, no activity was detected with the disaccharides D-lactose and D-cellobiose. In addition to catalysing the oxidation of primary alcohols, Gox has been reported to convert aldehydes to the corresponding carboxylates [38]. However, the aldehyde, glyoxal, turned over by glyoxal oxidase, also did not give activity with GlxA. In contrast, glycolaldehyde $\left(\mathrm{C}_{2} \mathrm{H}_{4} \mathrm{O}_{2}\right)$ the smallest molecule to contain both an aldehyde and a hydroxy group gave significant activity with GlxA that followed Michaelis-Menten kinetics (Figure 6B). From these data a $K_{\mathrm{m}}$ for glycolaldehyde of $115 \mathrm{mM}$ and a maximal aerobic turnover $\left(k_{\text {cat }}\right)$ of $0.14 \mathrm{~s}^{-1}$ was determined. The corresponding $k_{\text {cat }} / K_{\mathrm{m}}$ value of $1.22 \mathrm{M}^{-1} \cdot \mathrm{s}^{-1}$ enables a direct comparison with the two monosaccharides and glycerol, with glycolaldehyde reacting 100-times faster. A further possibility for the enzymatic role of GlxA is that the production of $\mathrm{H}_{2} \mathrm{O}_{2}$ is required for development rather than the substrate product. However, addition of sublethal concentrations of $\mathrm{H}_{2} \mathrm{O}_{2}$ to young cultures of both the wildtype (WT) and the $\triangle g l x A$ strains did not stimulate development (Supplementary Figure S3).

\section{GIXA is required for glycan synthesis at hyphal tips and morphogenesis}

To investigate a functional role of GlxA, we created a null-mutant in S. lividans. Consistent with previous work in S. coelicolor, our S. lividans glxA null-mutant has a bald phenotype (i.e. no aerial hyphae formation) on solid media, as is also the case for the cslA null-mutant $[18,20]$ (Figure 7A). Notably, the bald phenotype in the $g l x A$ and $c s l A$ null-mutants cannot be rescued upon addition of exogenous $\mathrm{Cu}$ (II) to the medium (Supplementary Figure S4) as has previously been reported for the $\Delta s c o$ nullmutant [7]. Furthermore, the glxA and cslA mutants have an identical morphology in liquid-grown cultures. Instead of the dense compact pellets seen in WT, the mutants grow with an open mycelium phenotype [4] (Figure 7A). The transcriptomes of the $g l x A$ and the $c s l A$ mutant as analysed by RNA-seq revealed increased expression of genes related to osmoprotection, indicating that the mutants suffer from osmotic stress (result not shown), and is in agreement with the observation of Liman et al. [18]. Excitingly, mycelial staining with CFW, which binds to $\beta$-(1-4) glycans indicated that the hyphal tips in both the $c s l A$ and $g l x A$ null mutants were no longer stained, in contrast with those of the WT strain (Figure 7B). This indicates that in addition to CslA, GlxA is required for glycan synthesis or attachment to hyphal tips.

\section{DISCUSSION}

Results from the present study reveal that S. lividans GlxA is a membrane-associated cuproenzyme required for the production or localization of the hyphal tip glycan. The spectroscopic properties, tertiary structure and enzymatic profile are all distinctly different 

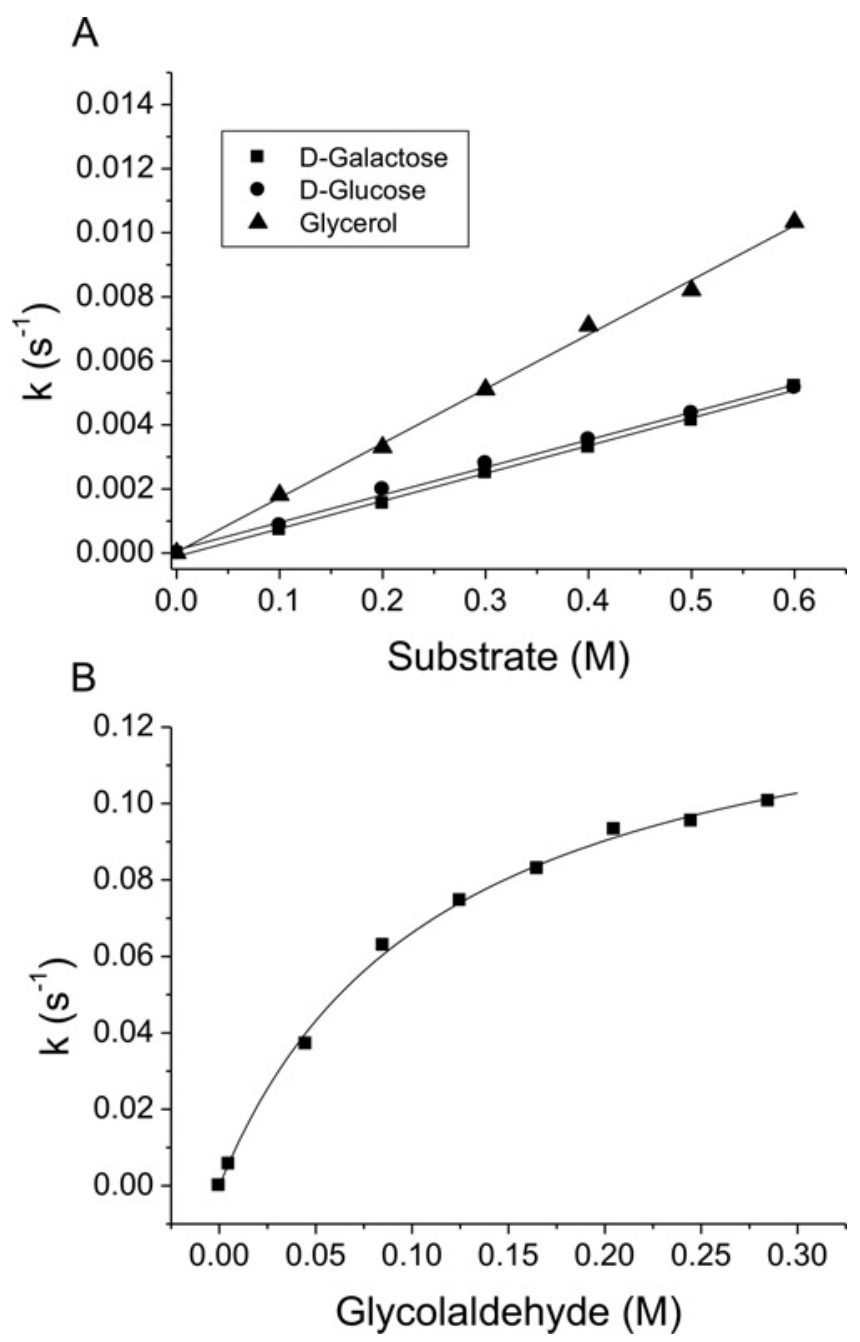

Figure 6 Enzyme activity of $S$. lividans GIXA

(A and B) Plots of turnover rates $(k)$ for GlxA with four different substrates $\left(25^{\circ} \mathrm{C}\right)$. In $(\mathbf{A})$, the line of best fit through the data points enables a second-order rate constant $\left(\mathrm{M}^{-1} \cdot \mathrm{S}^{-1}\right)$ to be determined. In (B) the data points have been fitted to the Michaelis-Menten equation to yield a $K_{\mathrm{m}}$ value and turnover $\left(k_{\text {cat }}\right)$ reported in the main text.

to its fungal counterpart Gox, thus making GlxA a new structural and functional member of the mononuclear $\mathrm{Cu}$ oxidase family.

From a spectroscopic perspective a number of features in GlxA stand out. In the $\mathrm{Cu}(\mathrm{II})-\mathrm{GlxA}$ EPR spectrum, the $\mathrm{g}_{\perp}$ component displays a hyperfine interaction of the electron spin with the $\mathrm{Cu}(\mathrm{II})$ ligands, giving rise to seven lines with relative intensities 1:4:8:10:8:4:1 yielding a $16 \mathrm{G}$ splitting (Figure 3C, inset). For $\mathrm{Cu}(\mathrm{II})$-Gox, a quintet line structure of relative intensities $1: 2: 3: 2: 1$ $[33,34]$ and a distance between the lines typical for a $\mathrm{N}$-hyperfine interaction (14-18 G) [39,40] consistent with two histidine ligands is observed. The source of the additional hyperfine splitting in GlxA is presently unclear and requires further investigation, but would suggest that differences in the electronic properties of the $\mathrm{Cu}$ (II) site in GlxA and Gox exist. A further anomaly in the spectroscopic properties between GlxA and Gox is in relation to the spectral observation of the Y-C• radical. EPR spectroscopy clearly indicates the presence of this radical (Figure 5) following addition of an oxidant to $\mathrm{Cu}$ (II)-GlxA. However, no perturbation of the $\mathrm{Cu}$ (II)-GlxA absorbance spectrum is observed as is the case on forming $\mathrm{Cu}(\mathrm{II})-\mathrm{Y}-\mathrm{C} \bullet$ in Gox. Furthermore, the $\mathrm{Cu}(\mathrm{II})-\mathrm{GlxA}$ optical spectrum does not resemble that of $\mathrm{Cu}$ (II)-Gox [11]. The co-ordination geometry of the $\mathrm{Cu}$ (II) site in both GlxA and Gox are identical, but as indicated by EPR could be electronically distinct with second sphere co-ordination contributing. In this respect, the most pertinent difference between GlxA and Gox is in the side chain orientation of $\operatorname{Trp}^{288}$ (Trp ${ }^{290}$ in Gox). In Gox, Trp ${ }^{290}$ has been proposed to provide an extended aromatic system with its indole ring stacking over the $\mathrm{Y}-\mathrm{C}$ bond, stabilizing the radical (Figure 8D) [13,41]. However, in GlxA Trp ${ }^{288}$ no longer has its indole ring stacking with the $\mathrm{Y}-\mathrm{C}$ cross-link, but instead has the benzene ring component $\pi-\pi$ stacking with the $\operatorname{Tyr}^{289} \mathrm{Cu}$ ligand (Figure 8D). In combination with a more insulated (buried) $\mathrm{Cu}$ site, the re-orientation of the tryptophan side chain may influence the electronic transitions of the $\mathrm{Cu}$ and the radical and result in the markedly different absorbance spectrum for GlxA compared with Gox.

The ability to generate three oxidation states in GlxA suggests an enzymatic function in S. lividans. However, none of the substrates tested exhibit activity with GlxA that is comparable to Gox (with the exception of D-glucose). This is exemplified with Dgalactose, the canonical monosaccharide substrate for Gox, where under aerobic conditions a $k_{\text {cat }} / K_{\mathrm{m}}$ value of $10^{-3} \mathrm{M}^{-1} \cdot \mathrm{s}^{-1}$ for GlxA is determined, which is a million times lower than for Gox [42] and is equivalent to Gox with D-glucose [43]. The most active substrate tested with GlxA, glycolaldehyde, has a $K_{\mathrm{m}}$ value (115 mM) comparable to Gox for D-galactose (70-80 mM), but a $k_{\text {cat }} / K_{\mathrm{m}}$ value $>4$-orders of magnitude lower. For Gox the turnover of glycoladehyde has been reported to be $75 \%$ of the D-galactose rate [44]. These slow turnover kinetics indicate that GlxA has very different substrate specificity compared with Gox, a likely consequence of gross structural differences.

The distinctness of the GlxA structure can be appreciated from the superposition with Gox in Figure 8(A). In Gox, the domain arrangement and surface flatness of the $\beta$-propeller domain (domain 2) contributes to a readily accessible $\mathrm{Cu}$ site and substrate-binding pocket (Figures $8 \mathrm{~B}$ and $8 \mathrm{C}$ ) [13]. This is not the case in GlxA, due to the positioning of domain 2 and the longer loop structures of the Kelch motifs. The more accessible nature of the $\mathrm{Cu}$ site in Gox is further apparent in the presence of an acetate ion (a buffer component of the crystallization solution) co-ordinating the $\mathrm{Cu}$ in the X-ray structure. Despite crystals of GlxA also being grown from acetate buffer, no evidence for an acetate ion, in or close, to the $\mathrm{Cu}$ site is observed, highlighting the protection afforded to the $\mathrm{Cu}$ site in GlxA. Furthermore, a very different structural arrangement of the substrate pocket in GlxA compared with Gox is apparent (Figure 8D). On one side of the Gox pocket, the side chains of residues $\mathrm{Arg}^{330}, \mathrm{Gln}^{406}$ and the $\mathrm{N} \varepsilon 1$ atom of $\operatorname{Trp}^{290}$ are posed to provide $\mathrm{H}$-bonds to the canonical D-galactose substrate, with the opposite side of the pocket formed by the aromatic residues, $\mathrm{Phe}^{194}$ and $\mathrm{Phe}^{464}$, creating an asymmetric polar/apolar substrate pocket. For GlxA the residues in the pocket, with the exception of $\operatorname{Trp}^{288}$, differ and are not spatially conserved (Figure 8D). Furthermore, Trp ${ }^{288}$ in GlxA, has its indole ring 'flipped' relative to $\operatorname{Trp}^{290}$ in Gox, making this orientation less favourable from a distance perspective for a substrate H-bond interaction in the pocket (Figure 8D) [15]. These differences serve to illustrate that despite the $\mathrm{Cu}$ co-ordination being similar, the substrate pockets between Gox and GlxA contain no conserved features. Therefore substrates displaying high turnover kinetics with Gox are unlikely to be optimally accommodated or positioned in GlxA enabling similar high turnover rates to be achieved.

The observations from the molecular genetics and CFW staining (Figure 7) indicate that GlxA is directly involved in the production or localization of the hyphal tip glycan. This is consistent with GlxA localization on the hyphal tip [18]. 
A
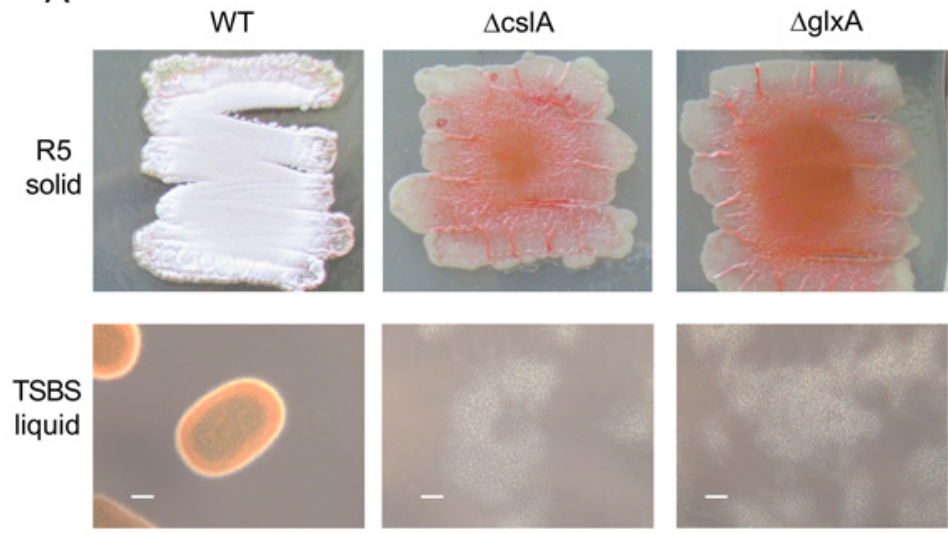

B

WT

$\Delta \mathrm{csI} A$
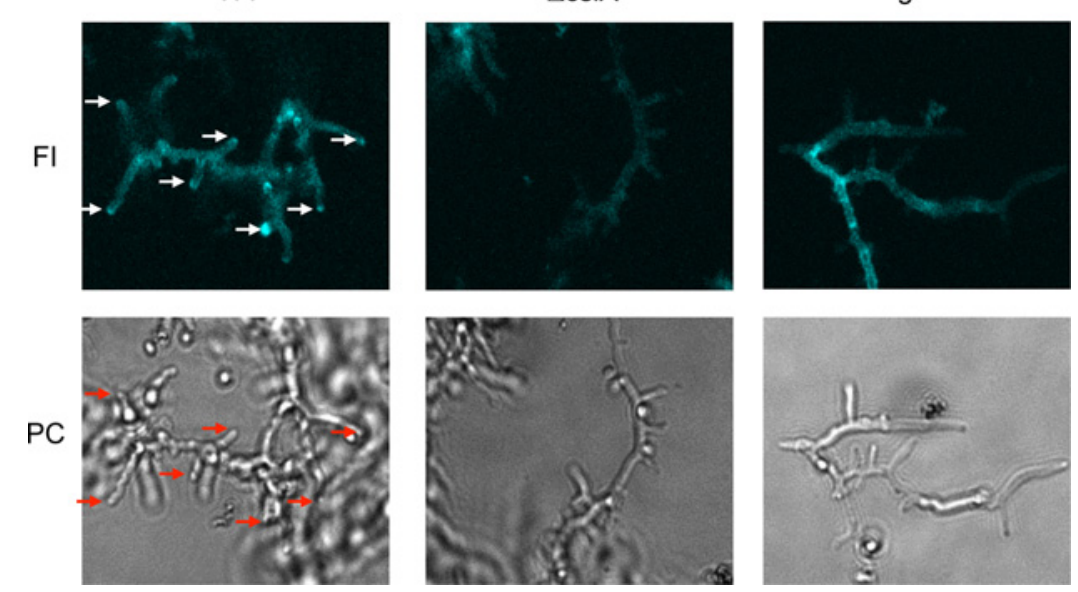

\section{Figure 7 Deletion of $c$ sIA or $g / x A$ in $S$. lividans leads to a block in development and abolishes pellet formation and glycan deposition}

(A) Growth on solid R5 medium and pellet formation and dispersed growth in TSBS $24 \mathrm{~h}$ liquid cultures. Scale-bar (white line) is $100 \mu \mathrm{m}$. (B) CFW staining in $24 \mathrm{~h}$ mNMMP standing cultures shown in fluorescence image (FI) and phase contrast brightfield (PC). Arrows indicate tip staining that is present in WT cultures but not in mutants.

A

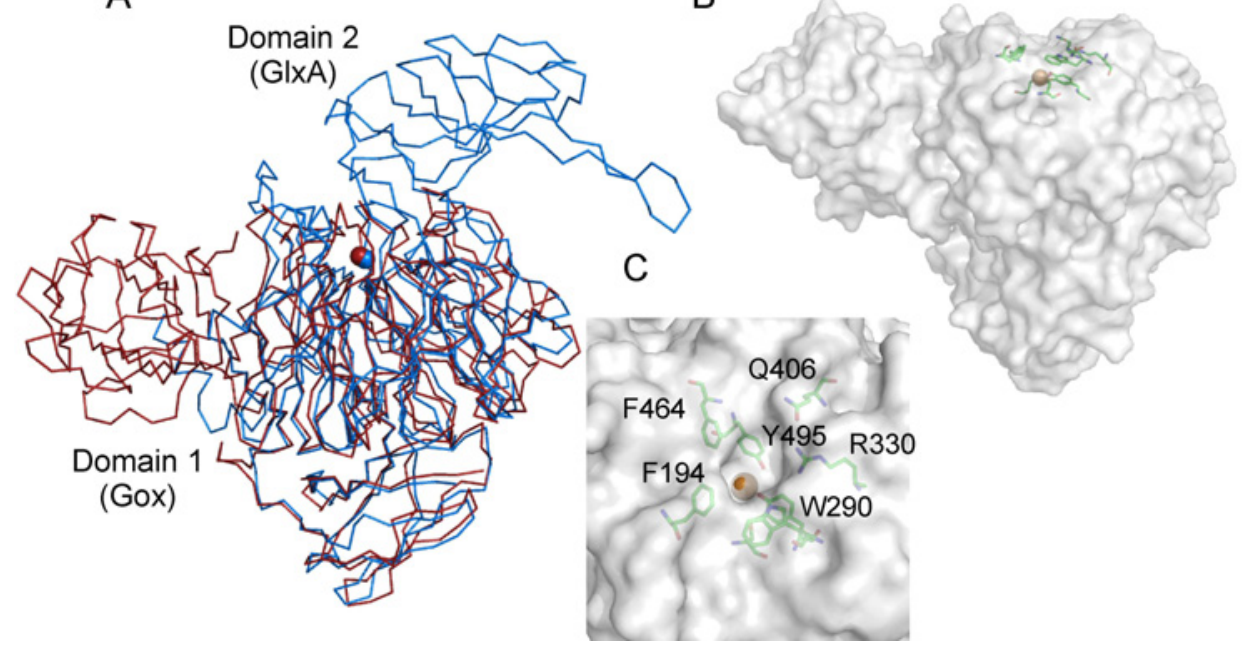

D

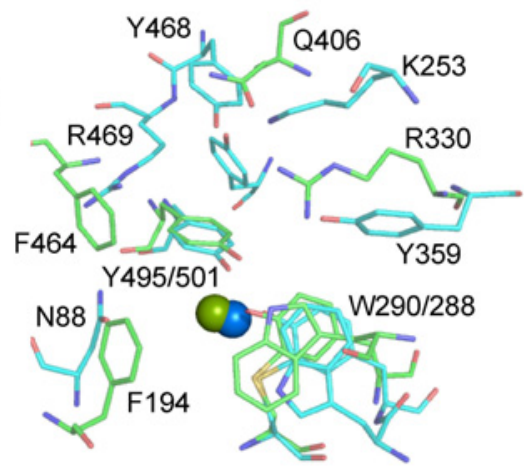

Figure 8 Structural comparison of GIxA with Gox (1gof) [13]

(A) Ribbon representation of a superposition of the $\mathrm{C} \alpha$ atoms of GIXA (blue) with Gox (red). The Cu ions are shown as spheres. (B) Surface representation of Gox, with location of the Cu ion (brown sphere) and residues in the substrate pocket indicated in sticks. (C) View of the substrate pocket looking down the 7-fold symmetry axis towards domain 3 of Gox. The location of the surface exposed $\mathrm{Tyr}^{495} \mathrm{Cu}$ ligand and the stacking Trp ${ }^{290}$ as well as residues forming the substrate pocket are shown in sticks, with the solvent exposed Cu represented as a sphere. (D) Superposition of the substrate pockets in GIXA (blue) and Gox (green). 
Synthesis of this glycan also requires CslA, which is a cellulose synthase-like enzyme encoded by the translationally-coupled gene upstream of $g l x A$. This glycan accumulates at apical sites during vegetative growth and is absent in the cslA mutant $[19,20,45]$ and also in the glxA mutant. The molecular identity of this glycan is not yet known, but we propose that GlxA acts to modify it through a two-electron oxidation process. It is tempting to speculate that the orientations of tunnels I and II, both connecting at the $\mathrm{Cu}$ site (Figure 4), could be a key structural feature utilized by GlxA in the oxidation of this glycan. A scenario whereby the nascent glycan produced by CslA is fed down into one of these tunnels, orientated in the substrate pocket, oxidized and then released through the other tunnel may be envisaged. From a biotechnology perspective the open mycelium structure of the $g l x A$ and $c s l A$ null-mutants in liquid-grown cultures is of interest for improvement in the utilization of S. lividans as an enzyme production host. It has been shown that a more fragmented and therefore also more open mycelium growth in Streptomyces increases enzyme production several fold $[4,46]$ and that the absence of other glycans result in a distinct morphology in liquid cultures [47].

Finally, the inability of the $\triangle g l x A$ phenotype to be rescued by $\mathrm{Cu}$ links GlxA to the $\mathrm{Cu}$ dependency of $S$. lividans (Supplementary Figure S4) and raises the question of how GlxA acquires $\mathrm{Cu}$. Our previous work has inferred that the $\mathrm{Cu}$ chaperone Sco delivers $\mathrm{Cu}$ to an $a a_{3}$-type $\mathrm{CcO}$ and also to an unidentified target, which is required to trigger aerial hyphae growth $[7,8]$. To date no other Sco target except $\mathrm{CcO}$ in either eukaryote or prokaryote species has been identified. The possibility therefore arises that the second Sco target in S. lividans is GlxA. Further experiments aimed at exploring whether this is the case are planned.

\section{AUTHOR CONTRIBUTION}

Jonathan Worrall, Erik Vijgenboom and Dennis Claessen conceived and designed the project. Amanda Chaplin cloned, overexpressed and purified recombinant GIXA, crystallized and solved the X-ray structure and performed all spectroscopic and enzymatic activity measurements. Marloes Petrus, Giulia Mangiameli, Dennis Claessen and Erik Vijgenboom carried out the molecular genetics, GIXA location and glycan detection. Michael Hough assisted with X-ray data collection and monitored the structural work. Dimitri Svistunenko helped interpret the EPR data. Peter Nicholls advised and assisted with enzymatic assays. Jonathan Worrall, Erik Vijgenboom and Dennis Claessen wrote the manuscript with contributions from all authors.

\section{ACKNOWLEDGEMENTS}

Diamond Light Source for access to beamline 103 (East of England Macromolecular Crystallography BAG, MX7461) and use of the JCSG Quality Control Server is acknowledged.

\section{FUNDING}

This work was supported by the University of Essex Silberrad Scholarship (to A.K.C.); the Dutch Applied Research Council [grant number 12957 (to D.C.)]; the Netherlands Organization for Scientific Research-Advanced Chemical Technologies for Sustainability (ACTS) [grant number 053.80.703EIB.08.013 (to E.V.)].

\section{REFERENCES}

1 Flärdh, K. and Buttner, M.J. (2009) Streptomyces morphogenetics: dissecting differentiation in a filamentous bacterium. Nat. Rev. Microbiol. 7, 36-49 CrossRef PubMed
2 Claessen, D., Rozen, D.E., Kuipers, O.P., Søgaard-Andersen, L. and van Wezel, G.P. (2014) Bacterial solutions to multicellularity: a tale of biofilms, filaments and fruiting bodies. Nat. Rev. Microbiol. 12, 115-124 CrossRef PubMed

3 Anne, J., Maldonado, B., Van Impe, J., Van Mellaert, L. and Bernaerts, K. (2012) Recombinant protein production and streptomycetes. J. Biotechnol. 158, 159-167 CrossRef PubMed

4 van Dissel, D., Claessen, D. and van Wezel, G.P. (2014) Morphogenesis of Streptomyces in submerged cultures. Adv. Appl. Microbiol. 89, 1-45 PubMed

5 Keijser, B.J., van Wezel, G.P., Canters, G.W., Kieser, T. and Vijgenboom, E. (2000) The ram-dependence of Streptomyces lividans differentiation is bypassed by copper. J. Mol. Microbiol. Biotechnol. 2, 565-574 PubMed

6 Fujimoto, M., Yamada, A., Kurosawa, J., Kawata, A., Beppu, T., Takano, H. and Ueda, K. (2012) Pleiotropic role of the Sco1/SenC family copper chaperone in the physiology of Streptomyces. Microb. Biotech. 5, 477-488 CrossRef

7 Blundell, K.L., Wilson, M.T., Svistunenko, D.A., Vijgenboom, E. and Worrall, J.A. (2013) Morphological development and cytochrome c oxidase activity in Streptomyces lividans are dependent on the action of a copper bound Sco protein. Open Biol. 3, 120163 CrossRef PubMed

8 Blundell, K.L., Hough, M.A., Vijgenboom, E. and Worrall, J.A. (2014) Structural and mechanistic insights into an extracytoplasmic copper trafficking pathway in Streptomyces lividans. Biochem. J. 459, 525-538 CrossRef PubMed

9 Worrall, J.A. and Vijgenboom, E. (2010) Copper mining in Streptomyces: enzymes, natural products and development. Nat. Prod. Rep. 27, 742-756 CrossRef PubMed

10 Cruz-Morales, P., Vijgenboom, E., Iruegas-Bocardo, F., Girard, G., Yanez-Guerra, L.A., Ramos-Aboites, H.E., Pernodet, J.L., Anne, J., van Wezel, G.P. and Barona-Gomez, F. (2013) The genome sequence of streptomyces lividans 66 reveals a novel tRNA-dependent peptide biosynthetic system within a metal-related genomic Island. Genome Biol. Evol. 5, 1165-1175 CrossRef PubMed

11 Whittaker, M.M. and Whittaker, J.W. (1988) The active site of galactose oxidase. J. Biol. Chem. 263, 6074-6080 PubMed

12 Whittaker, M.M. and Whittaker, J.W. (1990) A tyrosine-derived free radical in apogalactose oxidase. J. Biol. Chem. 265, 9610-9613 PubMed

13 Ito, N., Phillips, S.E., Stevens, C., Ogel, Z.B., McPherson, M.J., Keen, J.N., Yadav, K.D. and Knowles, P.F. (1991) Novel thioether bond revealed by a 1.7 A crystal structure of galactose oxidase. Nature 350, 87-90 CrossRef PubMed

14 Whittaker, M.M. and Whittaker, J.W. (1993) Ligand interactions with galactose oxidase: mechanistic insights. Biophys. J. 64, 762-772 CrossRef PubMed

15 Ito, N., Phillips, S.E., Yadav, K.D. and Knowles, P.F. (1994) Crystal structure of a free radical enzyme, galactose oxidase. J. Mol. Biol. 238, 794-814 CrossRef PubMed

16 Lee, Y.K., Whittaker, M.M. and Whittaker, J.W. (2008) The electronic structure of the Cys-Tyr $\left.{ }^{\star}\right)$ free radical in galactose oxidase determined by EPR spectroscopy. Biochemistry 47, 6637-6649 CrossRef PubMed

17 Avigad, G., Amaral, D., Asensio, C. and Horecker, B.L. (1962) The D-galactose oxidase of Polyporus circinatus. J. Biol. Chem. 237, 2736-2743 PubMed

18 Liman, R., Facey, P.D., van Keulen, G., Dyson, P.J. and Del Sol, R. (2013) A laterally acquired galactose oxidase-like gene is required for aerial development during osmotic stress in Streptomyces coelicolor. PLoS One 8, e54112 CrossRef PubMed

19 Petrus, M.L. and Claessen, D. (2014) Pivotal roles for Streptomyces cell surface polymers in morphological differentiation, attachment and mycelial architecture. Antonie Van Leeuwenhoek 106, 127-139 CrossRef PubMed

20 Xu, H., Chater, K.F., Deng, Z. and Tao, M. (2008) A cellulose synthase-like protein involved in hyphal tip growth and morphological differentiation in streptomyces. J. Bacteriol. 190, 4971-4978 CrossRef PubMed

21 Vijgenboom, E., Woudt, L.P., Heinstra, P.W., Rietveld, K., van Haarlem, J., van Wezel, G.P., Shochat, S. and Bosch, L. (1994) Three tuf-like genes in the kirromycin producer Streptomyces ramocissimus. Microbiology 140 (Pt 4), 983-998 CrossRef PubMed

22 Schneider, C.A., Rasband, W.S. and Eliceiri, K.W. (2012) NIH Image to ImageJ: 25 years of image analysis. Nat. Methods $\mathbf{9}, 671-675$ CrossRef PubMed

23 Kieser, T.B., M.J. Buttner, M.J. Chater and K.F. Hopwood, D.A. (2000) Pratcical Streptomyces Genetics. John Innes Foundation, Norwich, UK

24 van Keulen, G., Jonkers, H.M., Claessen, D., Dijkhuizen, L. and Wösten, H.A. (2003) Differentiation and anaerobiosis in standing liquid cultures of Streptomyces coelicolor. J. Bacteriol. 185, 1455-1458 CrossRef PubMed

25 Kozlikova, B., Sebestova, E., Sustr, V., Brezovsky, J., Strnad, O., Daniel, L., Bednar, D., Pavelka, A., Manak, M., Bezdeka, M. et al. (2014) CAVER Analyst 1.0: Graphic tool for interactive visualization and analysis of tunnels and channels in protein structures. Bioinformatics 30, 2684-2685 CrossRef PubMed

26 Petersen, T.N., Brunak, S., von Heijne, G. and Nielsen, H. (2011) SignalP 4.0: discriminating signal peptides from transmembrane regions. Nat. Methods 8, 785-786 CrossRef PubMed

27 Kall, L., Krogh, A. and Sonnhammer, E.L. (2004) A combined transmembrane topology and signal peptide prediction method. J. Mol. Biol. 338, 1027-1036 CrossRef PubMed 
28 Bagos, P.G., Tsirigos, K.D., Liakopoulos, T.D. and Hamodrakas, S.J. (2008) Prediction of lipoprotein signal peptides in Gram-positive bacteria with a Hidden Markov Model. J. Proteome Res. 7, 5082-5093 CrossRef PubMed

29 Sillitoe, I., Cuff, A.L., Dessailly, B.H., Dawson, N.L., Furnham, N., Lee, D., Lees, J.G., Lewis, T.E., Studer, R.A., Rentzsch, R. et al. (2013) New functional families (FunFams) in CATH to improve the mapping of conserved functional sites to $3 \mathrm{D}$ structures. Nucleic Acids Res. 41, D490-D498 CrossRef PubMed

30 Antholine, W.E. (2005) Low frequency EPR of Cu(II) in proteins. Biol. Mag. Res. 23, 417-454

31 Whittaker, M.M. and Whittaker, J.W. (2003) Cu(I)-dependent biogenesis of the galactose oxidase redox cofactor. J. Biol. Chem. 278, 22090-22101 CrossRef PubMed

32 Peisach, J. and Blumberg, W.E. (1974) Structural implications derived from the analysis of electron paramagnetic resonance spectra of natural and artificial copper proteins. Arch. Biochem. Biophys. 165, 691-708 CrossRef PubMed

33 Cleveland, L., Coffman, R.E., Coon, P. and Davis, L. (1975) An investigation of the role of the copper in galactose oxidase. Biochemistry 14, 1108-1115 CrossRef PubMed

34 Bereman, R.D. and Kosman, D.J. (1977) Stereoelectronic properties of metalloenzymes. 5. Identification and assignment of ligand hyperfine splittings in the electron spin resonance spectrum of galactose oxidase. J. Am. Chem. Soc. 99, 7322-7325 CrossRef PubMed

35 Chovancova, E., Pavelka, A., Benes, P., Strnad, 0., Brezovsky, J., Kozlikova, B., Gora, A., Sustr, V., Klvana, M., Medek, P. et al. (2012) CAVER 3.0: a tool for the analysis of transport pathways in dynamic protein structures. PLoS Comp. Biol. 8, e1002708 CrossRef

36 Whittaker, M.M., Kersten, P.J., Cullen, D. and Whittaker, J.W. (1999) Identification of catalytic residues in glyoxal oxidase by targeted mutagenesis. J. Biol. Chem. 274, 36226-36232 CrossRef PubMed

37 Whittaker, M.M. and Whittaker, J.W. (2006) Streptomyces coelicolor oxidase (SC02837p): a new free radical metalloenzyme secreted by Streptomyces coelicolor A3(2). Arch. Biochem. Biophys. 452, 108-118 CrossRef PubMed

38 Kelleher, F.M. and Bhavanandan, V.P. (1986) Re-examination of the products of the action of galactose oxidase. Evidence for the conversion of raffinose to 6"-carboxyraffinose. J. Biol. Chem. 261, 11045-11048 PubMed

Received 12 February 2015/27 May 2015; accepted 1Jun 2015

Published as BJ Immediate Publication 10 June 2015, doi:10.1042/BJ20150190
39 Berliner, L.J., Khramtsov, V., Fujii, H. and Clanton, T.L. (2001) Unique in vivo applications of spin traps. Free Rad. Biol. Med. 30, 489-499 CrossRef

40 Kirima, K., Tsuchiya, K., Sei, H., Hasegawa, T., Shikishima, M., Motobayashi, Y., Morita K., Yoshizumi, M. and Tamaki, T. (2003) Evaluation of systemic blood NO dynamics by EPR spectroscopy: $\mathrm{HbNO}$ as an endogenous index of NO. Am. J. Physiol. Heart Circ. Physiol. 285, H589-H596 CrossRef PubMed

41 Rogers, M.S., Tyler, E.M., Akyumani, N., Kurtis, C.R., Spooner, R.K., Deacon, S.E., Tamber, S., Firbank, S.J., Mahmoud, K., Knowles, P.F. et al. (2007) The stacking tryptophan of galactose oxidase: a second-coordination sphere residue that has profound effects on tyrosyl radical behavior and enzyme catalysis. Biochemistry 46, 4606-4618 CrossRef PubMed

42 Baron, A.J., Stevens, C., Wilmot, C., Seneviratne, K.D., Blakeley, V., Dooley, D.M., Phillips, S.E., Knowles, P.F. and McPherson, M.J. (1994) Structure and mechanism of galactose oxidase. The free radical site. J. Biol. Chem. 269, 25095-25105 PubMed

43 Sun, L., Bulter, T., Alcalde, M., Petrounia, I.P. and Arnold, F.H. (2002) Modification of galactose oxidase to introduce glucose 6-oxidase activity. ChemBioChem 3, 781-783 CrossRef PubMed

44 Arends, I.W. C.E., Gamez, P. and Sheldon, R.A. (2006) Green oxidation of alcohols using biomimetic Cu complexes and Cu enzymes as catalysts. Advs. Inorg. Chem. 58, 235-279

45 de Jong, W., Wösten, H.A., Dijkhuizen, L. and Claessen, D. (2009) Attachment of Streptomyces coelicolor is mediated by amyloidal fimbriae that are anchored to the cell surface via cellulose. Mol. Microbiol. 73, 1128-1140 CrossRef PubMed

46 van Wezel, G.P., Krabben, P., Traag, B.A., Keijser, B.J., Kerste, R., Vijgenboom, E., Heijnen, J.J. and Kraal, B. (2006) Unlocking Streptomyces spp. for use as sustainable industrial production platforms by morphological engineering. Appl. Environ. Microbiol. 72, 5283-5288 CrossRef PubMed

47 van Dissel, D., Claessen, D., Roth, M. and van Wezel, G.P. (2015) A novel locus for mycelial aggregation forms a gateway to improved Streptomyces cell factories. Microbial Cell Facts 14, 44 CrossRef

48 McNicholas, S., Potterton, E., Wilson, K.S. and Noble, M.E. (2011) Presenting your structures: the CCP4mg molecular-graphics software. Acta Crystallogr. D Biol. Crystallogr. 67, 386-394 CrossRef PubMed 